\title{
THE HOMOTOPY THEORY OF CYCLIC SETS ${ }^{1}$
}

BY

\author{
W. G. DWYER, M. J. HOPKINS AND D. M. KAN
}

\begin{abstract}
The aim of this note is to show that the homotopy theory of the cyclic sets of Connes [3] is equivalent to that of $\mathrm{SO}(2)$-spaces (i.e. spaces with a circle action) and hence to that of spaces over $K(Z, 2)$.
\end{abstract}

\section{Introduction.}

1.1 Summary. The aim of this note is to study the homotopy theory of the cyclic sets of Connes [3]. These are simplicial sets with some additional structure, or more precisely, diagrams of sets indexed by a category $\Lambda^{\text {op }}$ of cyclic operators which contains the category $\Delta^{\mathrm{op}}$ of simplicial operators as a subcategory.

After a few preliminaries (\$2), we construct a Quillen closed model category structure on the category of cyclic sets (\$3) and show that the resulting homotopy theory is equivalent to (\$4) that of $\mathrm{SO}(2)$-spaces (i.e. spaces with a circle action) as well as to (\$5) that of simplicial sets over the nerve of $\Lambda^{\mathrm{op}}$, which has the homotopy type of $K(Z, 2)$.

The first and the last of these results hold more generally, as their proofs depend only on certain properties (2.2 and 2.6) of the inclusion functor $j: \Delta^{\mathrm{op}} \rightarrow \Lambda^{\mathrm{op}}$. Some additional exampls are (\$6) a kind of covering $\mathbf{K}^{\mathrm{op}}$ of $\Lambda^{\mathrm{op}}$ and, for every simplicial group $G$, its flattening $\mathbf{b} G$. (The homotopy theory of $\mathbf{b} G$-sets (i.e. functors $\mathbf{b} G \rightarrow$ sets) is equivalent to the homotopy theory of simplicial sets over the classifying complex of $G$.)

1.2 Notation, terminology, etc. (i) The category $\Delta^{\mathrm{op}}$ of simplicial operators. This is the category with objects $\mathbf{0}, 1,2, \ldots$ and generating maps

$$
\begin{array}{ll}
d_{i}: \mathbf{n} \rightarrow \mathbf{n}-\mathbf{1}, & 0 \leqslant i \leqslant n, n>0, \\
s_{i}: \mathbf{n} \rightarrow \mathbf{n}+\mathbf{1}, & 0 \leqslant i \leqslant n,
\end{array}
$$

subject to the relations

$$
\begin{aligned}
d_{i} d_{j} & =d_{j-1} d_{i}, & & 0<j-i, \\
s_{j} s_{i} & =s_{i} s_{j-1}, & & 0<j-i, \\
d_{i} s_{j} & =s_{j-1} d_{i}: \mathbf{n} \rightarrow \mathbf{n}, & & 0<j-i \leqslant n, \\
& =\mathrm{id}, & & -1 \leqslant j-i \leqslant 0, \\
& =s_{j} d_{i-1}, & & j-i<-1 .
\end{aligned}
$$

Received by the editors November 9, 1984.

1980 Mathematics Subject Classification. Primary 55P15; Secondary 55U35.

${ }^{1}$ This research was in part supported by the National Science Foundation. 
(ii) The category $\Lambda^{\mathrm{op}}$ of cyclic operators. This is the category with objects $\mathbf{0}, \mathbf{1}, \mathbf{2}, \ldots$ and generating maps

$$
\begin{array}{ll}
d_{i}: n \rightarrow \mathbf{n}-\mathbf{1}, & 0 \leqslant i \leqslant n, n>0, \\
s_{i}: n \rightarrow \mathbf{n}+\mathbf{1}, & 0 \leqslant i \leqslant n+1,
\end{array}
$$

subject to the above relations $(*)$ as well as the cyclic relations

$$
\left(d_{0} s_{n+1}\right)^{n+1}=\mathrm{id}: \mathbf{n} \rightarrow \mathbf{n}, \quad n \geqslant 0 .
$$

Note that, except for these cyclic relations, there is no relation involving the first face operator and last degeneracy operator, i.e. $d_{0} s_{n+1} \neq s_{n} d_{0}: \mathbf{n} \rightarrow \mathbf{n}$.

(iii) The categories $\mathbf{S}$ of simplicial sets (i.e. $\Delta^{\mathrm{op}}-$ sets) and $\mathbf{S}^{c}$ of cyclic sets (i.e. $\Lambda^{\mathrm{op}}$-sets $)$. These are the categories which have as objects the functions $\Delta^{\mathrm{op}} \rightarrow$ sets and $\Lambda^{\mathrm{op}} \rightarrow$ sets, respectively, and as maps the natural transformations between them. A cyclic set $X$ is thus a simplicial set together with an extra degeneracy map $s_{n+1}: X_{n} \rightarrow X_{n+1}$ in each dimension $n \geqslant 0$, with the cyclic property that $\left(d_{0} s_{n+1}\right)^{n+1}$ $=$ id: $X_{n} \rightarrow X_{n}$. However, in general, $d_{0} s_{n+1} \neq s_{n} d_{0}: X_{n} \rightarrow X_{n}$.

The categories $\mathbf{S}$ and $\mathbf{S}^{c}$ are connected by the rather useful forgetful functor $j^{*}$ : $\mathbf{S}^{c} \rightarrow \mathbf{S}$, induced by the inclusion functor $j: \Delta^{\mathrm{op}} \rightarrow \Lambda^{\mathrm{op}}$.

(iv) The category $\mathbf{T}^{c}$ of $\mathrm{SO}(2)$-spaces and the forgetful functor $u: \mathbf{T}^{c} \rightarrow \mathbf{T}$. We denote by $\mathbf{T}$ the category of topological spaces, by $\mathbf{T}^{c}$ the category of $\mathrm{SO}(2)$-spaces, i.e. topological spaces with a continuous $\mathrm{SO}(2)$-action, and by $u: \mathbf{T}^{c} \rightarrow \mathbf{T}$ the functor which sends each $\mathrm{SO}(2)$-space to its underlying topological space.

2. The standard cyclic sets $\Lambda[n]$. This is a brief discussion of the standard cyclic sets $\Lambda[n]$ (the cyclic analogs of the standard simplicial sets $\Delta[n]$ ), much of which is explicit or implicit in [8].

2.1 The standard cyclic sets in $\Lambda[n]$. For every integer $n \geqslant 0$, the standard cyclic set $\Lambda[n]$ is given by

$$
\Lambda[n]=\operatorname{hom}^{\Lambda^{\mathrm{op}}}(\mathbf{n},-): \Lambda^{\mathrm{op}} \rightarrow \text { sets. }
$$

It has the universal property that, for every cyclic set $X$ and every $n$-simplex $x \in X$, there is a unique map (1.2(iii)) $c_{x}: \Lambda[n] \rightarrow X \in \mathbf{S}^{c}$ such that $c_{x} i_{n}=x$ (where $i_{n} \in \Lambda[n]$ denotes the generating $n$-simplex, i.e. the identity map of $\left.\mathbf{n}\right)$. This gives rise to a natural isomorphism

$$
\operatorname{hom}^{\mathbf{S}^{c}}(\Lambda[n], X) \approx \operatorname{hom}^{\mathbf{S}}\left(\Delta[n], j^{*} X\right) \approx X_{n} .
$$

The $\Lambda[n]$ and the maps between them form a $\Lambda$-diagram of cyclic sets, i.e. a functor $\Lambda[-]: \Lambda \rightarrow \mathbf{S}^{c}$, with the following property:

2.2 Proposition. In the induced (1.2(iii)) diagram of simplicial sets $j^{*} \Lambda[-]: \Lambda \rightarrow \mathbf{S}$, all maps are weak (homotopy) equivalences.

2.3 The cyclic sets $\Lambda[n, k]$. As in the simplicial theory [2, Chapter VIII, 3.3] we need the cyclic subsets $\Lambda[n, k] \subset \Lambda[n](0 \leqslant k \leqslant n)$ which are spanned by the $(n-1)$-simplices $d_{i} i_{n}(0 \leqslant i \leqslant n, i \neq k)$, which clearly give rise to natural isomorphisms

$$
\operatorname{hom}^{\mathbf{s}}(\Lambda[n, k], X) \approx \operatorname{hom}^{\mathbf{s}}\left(\Delta[n, k], j^{*} X\right), \quad 0 \leqslant k \leqslant n
$$


Moreover, it is not difficult to prove (using 2.2)

2.4 Proposition. The inclusions $\Lambda[n, k] \rightarrow \Lambda[n] \in \mathbf{S}^{c},(0 \leqslant k \leqslant n)$ induce weak equivalences $j^{*} \Lambda[n, k] \rightarrow j^{*} \Lambda[n] \in \mathbf{S}$.

2.5 The cyclic sets $\dot{\Lambda}[n]$. Also useful are the cyclic subsets $\dot{\Lambda}[n] \subset \Lambda[n](n>0)$ spanned by the $(n-1)$-simplices $d_{i} i_{n}(0 \leqslant i \leqslant n)$. They give rise to natural isomorphisms

$$
\operatorname{hom}^{\mathbf{s}}(\dot{\Lambda}[n], X) \approx \operatorname{hom}^{\mathbf{s}}\left(\dot{\Delta}[n], j^{*} X\right), \quad n>0 .
$$

Moreover, each $\dot{\Lambda}[n]$ is closely related to the direct limit $\partial \Lambda[n]$ of the diagram in $\mathbf{S}^{c}$ which consists of

(i) for every integer $i$ with $0 \leqslant i \leqslant n$, a copy $\Lambda[n]_{i}$ of $\Lambda[n-1]$, and

(ii) for every pair of integers $(i, j)$ with $0 \leqslant i<j \leqslant n$, a copy $\Lambda[n]_{i, j}$ of $\Lambda[n-2]$ together with a pair of maps

$$
\Lambda[n]_{i} \stackrel{c_{d_{j-1} i_{n-1}}^{\leftarrow}}{\leftarrow} \Lambda[n]_{i, j} \stackrel{c_{d_{i} i_{n-1}}}{\rightarrow} \Lambda[n]_{j}
$$

and which has the following nontrivial property:

2.6 Proposition. For each integer $n>0$, the obvious map $\partial \Lambda[n] \rightarrow \dot{\Lambda}[n] \in \mathbf{S}^{c}$ is an isomorphism.

Propositions 2.2 and 2.6 follow readily from

2.7 Proposition. Let $\mathrm{SO}(2) \times|\Delta[-1]|: \Delta \rightarrow \mathrm{T}^{c}(1.2(\mathrm{iv}))$ denote the obvious functor which sends $\mathbf{n}(n \geqslant 0)$ to the product of $\mathrm{SO}(2)$ and the geometric realization of $\Delta[n]$, with $\mathrm{SO}(2)$ acting on the left on itself and trivially on $|\Delta[n]|$. Then there exists a functor $M: \Lambda \rightarrow \mathbf{T}^{c}$ such that the following diagram commutes up to natural equivalences:

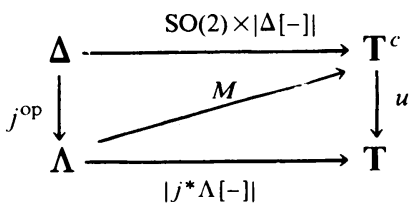

In particular, $\left|j^{*} \Lambda[n]\right|$ is homeomorphic to $\mathrm{SO}(2) \times|\Delta[n]|(n \geqslant 0)$.

As, for $X \in \mathbf{S}^{c}$, the geometric realization $\left|j^{*} X\right|$ can be expressed as a quotient of the disjoint union $\amalg_{n} X_{n} \times\left|j^{*} \Lambda[n]\right|$ by the obvious identifications, Proposition 2.7 readily implies

2.8 Proposition. There is a functor $L^{c}: S^{c} \rightarrow T^{c}$ such that the following diagram commutes up to a natural equivalence:

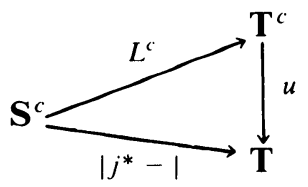


It thus remains to give a

2.9 Proof of Proposition 2.7. Consider the "twisted" product $\Delta[1] \times{ }_{t} \Delta[n] \in \mathbf{S}$ which has as $k$-simplices $(k \geqslant 0)$ the $(k+1)$-tuples of pairs of integers

$$
\left(\left(0, i_{1}\right), \ldots,\left(0, i_{a}\right),\left(1, j_{1}\right), \ldots,\left(1, j_{b}\right)\right)
$$

such that $0 \leqslant j_{1} \leqslant \cdots \leqslant j_{b} \leqslant i_{1} \leqslant \cdots \leqslant i_{a} \leqslant n$ and $a, b \geqslant 0$, with the obvious faces and degeneracies. Then there is a homeomorphism between the geometric realizations

$$
|\Delta[1] \times, \Delta[n]| \approx|\Delta[1] \times \Delta[n]|
$$

which is natural in $n$. As $j^{*} \Lambda[n]$ is exactly the simplicial set obtained from $\Delta[1] \times_{t}$ $\Delta[n]$ by identifying the simplices

$$
\left(\left(0, i_{1}\right), \ldots,\left(0, i_{a}\right)\right) \text { and }\left(\left(1, i_{1}\right), \ldots,\left(1, i_{a}\right)\right)
$$

for every sequence of integers $\left(i_{1}, \ldots, i_{a}\right)$ with $0 \leqslant i_{1} \leqslant \cdots \leqslant i_{a} \leqslant n$, the above homeomorphism induces a homeomorphism $\left|j^{*} \Lambda[n]\right| \approx \operatorname{SO}(2) \times|\Delta[n]|$ and the desired result now follows readily.

3. A homotopy theory for cyclic sets. In Theorem 3.1 below we turn the category $\mathbf{S}^{c}$ of cyclic sets (see subsection 1.2) into a closed model category in the sense of Quillen, i.e. [2, p. 241] we define notions of weak equivalence, fibration and cofibration such that the following five axioms are satisfied:

CM1. The category is closed under finite and direct and inverse limits.

CM2. If $f$ and $g$ are maps such that $g f$ is defined and if two of $f, g$ and gf are weak equivalences, then so is the third.

CM3. If $f$ is a retract of $g$ (i.e. if there are, in the category of maps, maps $a: f \rightarrow g$ and $b: g \rightarrow f$ such that $b a=\mathrm{id}_{f}$ ) and $g$ is a weak equivalence, a fibration or $a$ cofibration, then so is $f$.

CM4. Given a commutative solid arrow diagram

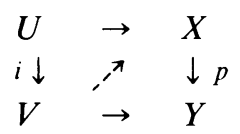

in which $i$ is a cofibration, $p$ is a fibration, and either $p$ or $i$ is a weak equivalence, then the dotted arrow exists (and one says that $i$ has the left lifting property with respect to $p$, or equivalently, that $p$ has the right lifting property with respect to $i$ ).

CM5. Any map $f$ can be factored in two ways:

(i) $f=p i$, where $i$ is a cofibration and $p$ is a trivial fibration (i.e. a fibration as well as a weak equivalence), and

(ii) $f=p i$, where $p$ is a fibration and $i$ is a trivial cofibration (i.e. a cofibration as well as a weak equivalence).

3.1 THEOREM. The category $\mathbf{S}^{c}$ of cyclic sets admits a closed model category structure in which a map $X \rightarrow Y \in \mathbf{S}^{c}$ is a weak equivalence or a fibration whenever the induced (1.2) map $^{*} X \rightarrow j^{*} Y \in \mathbf{S}$ is so, and in which the cofibrations are (see also 3.4) the retracts of the (possibly transfinite) compositions of cobase extensions of the inclusions $\Lambda[n] \rightarrow \dot{\Lambda}[n](n \geqslant 0)$. 
To prove this we first note that 2.3 and 2.5 imply

3.2 Proposition. A map in $\mathbf{S}^{c}$ is a fibration iff it has the right lifting property with respect to the inclusions $\Lambda[n, k] \rightarrow \Lambda[n](0 \leqslant k \leqslant n)$.

3.3 Proposition. A map in $\mathbf{S}^{c}$ is a trivial fibration iff it has the right lifting property with respect to the inclusions $\dot{\Lambda}[n] \rightarrow \Lambda[n](n \geqslant 0)$.

Proof of Theorem 3.1. Verification of CM1, CM2, CM3 and the first part of CM4 is easy. To prove CM5 one combines the small object argument of [9, Chapter II, §3] with 3.3 or 2.4 and 3.2. This proof of CM5 implies that a trivial cofibration $U \rightarrow V$ admits a factorization $U \rightarrow V^{\prime} \rightarrow V$ in which (by construction) the map $U \rightarrow V^{\prime}$ is a trivial cofibration which has the left lifting property with respect to the fibrations and $V^{\prime} \rightarrow V$ is a (necessarily trivial) fibration. The remaining part of CM4 now follows from the fact that the map $U \rightarrow V$ is (easily seen to be) a retract of the map $U \rightarrow V^{\prime}$.

We end with some alternate descriptions of the cofibrations and the weak equivalences.

3.4 Cofibrations in $\mathbf{S}^{c}$. Call a map $X \rightarrow Y \in \mathbf{S}^{c}$ free if it is 1-1 and if, for every integer $n \geqslant 0$, the group of automorphisms of $\mathbf{n}$ (which is cyclic of order $n+1$ ) acts freely on the $n$-simplices of $Y$ which are not in the image of $X$. Clearly the inclusions $\Lambda[n, k] \rightarrow \Lambda[n]$ and $\Lambda[n] \rightarrow \Lambda[n]$ are free and so are their cobase extensions. In fact one readily verifies

3.5 Proposition. The free maps in $\mathbf{S}^{c}$ are exactly the (possibly transfinite) compositions of cobase extensions of the inclusions $\dot{\Lambda}[n] \rightarrow \Lambda[n](n \geqslant 0)$. Hence a map in $\mathbf{S}^{c}$ is a cofibration iff it is a free map.

3.6 Weak equivalences in $\mathbf{S}^{c}$. Given a cyclic set $X$, i.e. a functor $X: \Lambda^{\text {op }} \rightarrow$ sets, one can compose it with the inclusion functor $i$ : sets $\rightarrow \mathbf{S}$ and take the homotopy direct limit [2, Chapter XIII] holim $^{\Lambda^{\text {op }}} i X \in \mathbf{S}$. Then one has

3.7 Proposition $A$ map $X \rightarrow Y \in \mathbf{S}^{c}$ is a weak equivalence iff the induced map $\operatorname{holim}^{\Lambda^{\text {op }}} i X \rightarrow \operatorname{holim}^{\Lambda^{\text {op }}}$ iY $\in \mathbf{S}$ is so.

Proof. Let $\mathbf{X}$ be the category which has as objects the simplices of $X$ and as maps the cyclic operators between them. Then the nerve $N \mathbf{X}$ is clearly naturally isomorphic to holim ${ }^{\Lambda^{\mathrm{p}}} i X$. Similarly, if $j^{*} \mathbf{X} \subset \mathbf{X}$ is the subcategory consisting of the simplicial operators (the objects remain the same), then the nerve $N^{*} \mathbf{X}$ is naturally isomorphic to holim ${ }^{\Lambda^{\text {op }}} i j^{*} \mathbf{X}$. Moreover, by [2, Chapter XII, 4.3] the latter simplicial set is naturally weakly equivalent to $j^{*} X$. The under categories of the inclusion functor $j^{*} \mathbf{X} \rightarrow \mathbf{X}$ are readily verified to be isomorphic to the under categories $\mathbf{n} \downarrow j$ of the inclusion functor $j: \Delta^{\mathrm{op}} \rightarrow \Lambda^{\mathrm{op}}$ and, by the above arguments, the nerves of the latter are naturally isomorphic to holim ${ }^{\Delta^{\text {op }}} i j^{*} \Lambda[n]$ and hence naturally weakly equivalent to $j^{*} \Lambda[n]$. The desired result now follows immediately from 2.2 and Quillen's theorem $B[10$, p. 97]. 
4. Comparison with $\mathrm{SO}(2)$-spaces. In order to show that the homotopy theory of cyclic sets of $\S 3$ is equivalent, in the strong sense of $[5, \S 5]$, to the homotopy theory of $\mathrm{SO}(2)$-spaces (resulting from its usual model category structure (see Theorem 4.1)), we verify in Theorem 4.2 the existence of a pair of adjoint functors

$$
L^{c}:(\text { cyclic sets }) \leftrightarrow(\mathrm{SO}(2) \text {-spaces }): R^{c}
$$

satisfying the equivalence conditions of Quillen [9, Chapter I, Theorem 3]:

EQ1. The left adjoint $L^{c}$ sends cofibrations into cofibrations and weak equivalences between cofibrant objects into weak equivalences.

EQ2. The right adjoint $R^{c}$ sends fibrations into fibrations and weak equivalences between the fibrant objects into weak equivalences.

EQ3. For every cofibrant cyclic set $X$ and every fibrant $\mathrm{SO}(2)$-space $Y$, a map $X \rightarrow R^{c} Y$ is a weak equivalence iff its adjoint $L^{c} X \rightarrow Y$ is so.

First we recall from [6, 1.2 and 2.2]

4.1 THEOREM. The category $\mathbf{T}^{c}$ of $\mathrm{SO}(2)$-spaces (see subsection 1.2) admits a closed model category structure in which a map $X \rightarrow Y \in \mathbf{T}^{c}$ is a weak equivalence or $a$ fibration whenever the underlying map of topological spaces $u X \rightarrow u Y \in T$ is a weak homotopy equivalence or a Serre fibration, and in which the cofibrations are the retracts of the (possibly transfinite) compositions of cobase extensions of the inclusions $\mathrm{SO}(2) \times$ $|\dot{\Delta}[n]| \rightarrow \mathrm{SO}(2) \times|\Delta[n]|(n \geqslant 0)$.

In view of Proposition 2.8 one can then formulate

4.2 TheOREM. The functor $L^{c}: \mathbf{S}^{c} \rightarrow \mathbf{T}^{c}$ has as right adjoint the functor $R^{c}=$ $\operatorname{hom}\left(L^{c} \Lambda[-],-\right): \mathbf{T}^{c} \rightarrow \mathbf{S}^{c}$. Moreover, this pair of adjoint functors satisfies the (above) equivalence conditions of Quillen.

4.3 Corollary. The categories $\mathbf{S}^{c}$ and $\mathbf{T}^{c}$ have equivalent homotopy theories in the strong sense that [5] their simplicial localizations with respect to the weak equivalences are weakly equivalent in the sense of $[5, \S 2]$.

Proof. One verifies successively and without much difficulty the following properties:

(i) the adjointness;

(ii) $L^{c}$ preserves weak equivalences;

(iii) for every object $Y \in \mathbf{T}^{c}$, the simplicial set $j^{*} R^{c} Y$ is just the singular complex of the underlying topological space $u Y$;

(iv) $R^{c}$ preserves weak equivalences and fibrations;

(v) $L^{c}$ preserves cofibrations (use (iv) and adjointness);

(vi) for every object $Y \in \mathbf{T}^{c}$, the underlying map $u L^{c} R^{c} Y \rightarrow u Y$ of the adjunction map is the usual adjunction map from the geometric realization of the singular complex of $u Y$ back to $u Y$;

(vii) EQ3 is satisfied.

5. Comparison with simplicial sets over $K(Z, 2)$. As the homotopy theory of $\mathrm{SO}(2)$-spaces is (well known to be [4]) equivalent to that of simplicial sets over $K(Z, 2)$, Theorem 4.2 implies that the same holds for the homotopy theory of cyclic 
sets of $\S 3$. We will now give a direct proof of this result (Theorem 5.1) which only uses the fact that the functor $j: \Delta^{\mathrm{op}} \rightarrow \Lambda^{\mathrm{op}}$ has the properties described in Propositions 2.2 and 2.6.

To formulate Theorem 5.1, let $N \Lambda^{\text {op }}$ be the nerve of $\Lambda^{\text {op }}$ (which [3] is weakly equivalent to $K(Z, 2)$ ), let the category $\mathbf{S} / N \Lambda^{\text {op }}$ of simplicial sets over $N \Lambda^{\text {op }}$ have the closed model category structure induced by the usual one on $\mathbf{S}[\mathbf{9}$, Chapter II], let $*$ : $\Lambda^{\mathrm{op}} \rightarrow$ sets $\in \mathbf{S}^{\mathrm{c}}$ be the terminal object and let $L^{\Lambda^{\mathrm{op}}}: \mathbf{S}^{\mathrm{c}} \rightarrow \mathbf{S} / N \Lambda^{\mathrm{op}}$ be the functor which sends an object $X \in \mathbf{S}^{\mathrm{c}}$ to the map (see 3.6)

$$
\stackrel{\operatorname{holim}^{\Lambda^{\mathrm{op}}}}{\longrightarrow} i X \rightarrow \operatorname{holim}^{\Lambda^{\mathrm{op}}} i^{*}=N \Lambda^{\mathrm{op}} \text {. }
$$

Then one has

5.1 THEOREM. The functor $L^{\Lambda^{\text {op }}}: \mathbf{S}^{c} \rightarrow \mathbf{S} / N \Lambda^{\text {op }}$ has as right adjoint the functor

$$
R^{\Lambda^{\mathrm{op}}}=\operatorname{hom}\left(L^{\Lambda^{\mathrm{op}}} \Lambda[-],-\right): \mathbf{S} / N \Lambda^{\mathrm{op}} \rightarrow \mathbf{S}^{\mathrm{c}} .
$$

Moreover, this pair of adjoint functors satisfies the (see §4) equivalence conditions of Quillen.

5.2 Corollary. The categories $\mathbf{S}$ and $\mathbf{S} / N \Lambda^{\mathrm{op}}$ have equivalent homotopy theories in the strong sense that [5] their simplicial localizations with respect to the weak equivalences are weakly equivalent in the sense of $[5, \S 2]$.

Proof. The adjointness is obvious and Proposition 3.7 implies that a map $X \rightarrow Y \in \mathbf{S}^{c}$ is a weak equivalence iff the induced map $L^{\Lambda^{o p}} X \rightarrow L^{\Lambda^{o p}} Y \in \mathbf{S} / N \Lambda^{\text {op }}$ is so. Moreover, $L^{\Lambda^{\text {op }}}$ preserves cofibrations and hence it follows from the adjointness that $R^{\Lambda^{\circ p}}$ preserves fibrations and trivial fibrations. By [1, 1.2 and 1.3], this implies $\mathrm{EQ} 2$. Finally, to prove EQ3, it suffices to show that, for every fibrant object $Y \in \mathbf{S} / N \Lambda^{\text {op }}$, the adjunction map $L^{\Lambda^{\mathrm{op}}} R^{\Lambda^{\mathrm{op}}} Y \rightarrow Y \in \mathbf{S} / N \Lambda^{\mathrm{op}}$ is a weak equivalence.

To do this let $Y^{\prime}=(N j)^{*} Y \in \mathbf{S} / N \Delta^{\mathrm{op}}$ be the pull back over $N j: N \Delta^{\mathrm{op}} \rightarrow N \Lambda^{\mathrm{op}}$ and let

$$
L^{\Delta^{\mathrm{op}}}: \mathbf{S} \leftrightarrow \mathbf{S} / N \Delta^{\mathrm{op}}: R^{\Delta^{\mathrm{op}}}
$$

be the pair of adjoint functors analogous to $L^{\Lambda^{\text {op }}}$ and $R^{\Lambda^{\text {op }}}$. Then it is not difficult to verify that the adjunction map $L^{\Delta^{\circ p}} R^{\Delta^{\circ p}} Y^{\prime} \rightarrow Y^{\prime} \in \mathrm{S} / N \Delta^{\text {op }}$ is a weak equivalence and that this map admits an obvious factorization $L^{\Delta^{\mathrm{op}}} R^{\Delta^{\mathrm{op}}} Y^{\prime} \rightarrow L^{\Delta^{\mathrm{op}} j^{*}} R^{\Lambda^{\mathrm{op}}} Y \rightarrow Y^{\prime}$. Using the argument of Proposition 3.7 and the fact that $Y \in \mathbf{S} / N \Lambda^{\mathrm{op}}$ is fibrant, one now proves that the adjunction map $L^{\Lambda^{\mathrm{op}}} R^{\Lambda^{\mathrm{op}}} Y \rightarrow Y \in \mathrm{S} / N \Lambda^{\text {op }}$ is a weak equiva-

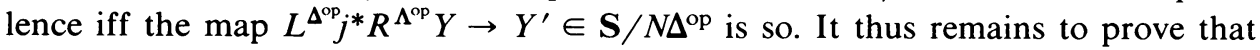
the map $L^{\Delta^{\mathrm{op}}} R^{\Delta^{\mathrm{op}}} Y^{\prime} \rightarrow L^{\Delta^{\mathrm{op}} j^{*}} R^{\Lambda^{\mathrm{op}}} Y \in \mathrm{S} / N \Delta^{\mathrm{op}}$ is a weak equivalence and to do this one observes $\left[4\right.$, §5] that the obvious maps holim ${ }^{\Delta^{\text {op }}} i \Delta[n] \rightarrow \operatorname{holim}^{\Lambda^{\text {op }}} i \Lambda[n] \in \mathbf{S}$ $(n \geqslant 0)$ are weak equivalences and that the $\Delta$-diagram $L^{\Lambda^{\mathrm{op}}} \Lambda[-] \circ j^{\mathrm{op}:} \Delta \rightarrow \mathbf{S} / N \Lambda^{\mathrm{op}}$ is a cosimplicial resolution [5, 4.3]. To prove this last statement one notes that

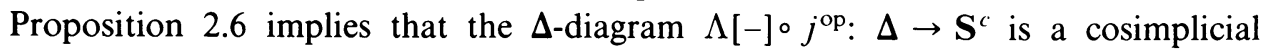
resolution and that the functor $L^{\Lambda^{\text {op }}}$ satisfies EQ1 and commutes with the direct limits in question.

5.3 REMARK. The last step in the above proof is the only place where we used Proposition 2.6. 
6. A generalization to other categories under $\Delta^{\mathrm{op}}$. As mentioned in 1.1 , Theorems 3.1 and 5.1 hold more generally, as their proofs only use the fact that the functor $j$ : $\Delta^{\mathrm{op}} \rightarrow \Lambda^{\mathrm{op}}$ has the properties described in Propositions 2.2 and 2.6. In order to formulate these generalizations, we start with a brief discussion of

6.1 Categories under $\Delta^{\mathrm{op}}$. Let $\Sigma$ be a small category and let $j: \Delta^{\mathrm{op}} \rightarrow \Sigma^{\mathrm{op}}$ be a functor. For every integer $n \geqslant 0$, one can then define the standard $\Sigma^{\mathrm{op}}$-set $\Sigma[n]$ by

$$
\Sigma[n]=\operatorname{hom}^{\Sigma^{\mathrm{op}}}(j \mathbf{n},-): \Sigma^{\mathrm{op}} \rightarrow \text { sets. }
$$

Clearly, these $\Sigma^{\text {op }}$-sets give rise to a $\Sigma$-diagram $\Sigma[-]: \Sigma \rightarrow \Sigma^{\text {op }}$-sets of $\Sigma^{\text {op }}$-sets as well as an induced $\Sigma$-diagram $j^{*} \Sigma[-]: \Sigma \rightarrow \mathbf{S}$ of simplicial sets. One can also consider the $\Sigma^{\mathrm{op}}$-subset $\dot{\Sigma}[n] \subset \Sigma[n]$ spanned by the "faces" of the generating element, i.e. the identity map of $j \mathbf{n}$, and the $\Sigma^{\text {op }}$-set $\partial \Sigma[n]$, defined by means of a direct limit as in 2.5.

We will assume that the functor $j: \Delta^{\mathrm{op}} \rightarrow \Sigma^{\mathrm{op}}$ satisfies one or both of the following conditions:

(i) In the diagram of simplicial sets $j^{*} \Sigma[-]: \Sigma \rightarrow \mathbf{S}$, all maps are weak equivalences.

(ii) For every integer $n>0$, the obvious map $\partial \Sigma[n] \rightarrow \dot{\Sigma}[n] \in \Sigma^{\mathrm{op}}$-sets is an isomorphism.

The arguments of the proof of Theorem 3.1 then yield

6.2 THEOREM. Let $\Sigma$ be a small category and let: $\Delta^{\mathrm{op}} \rightarrow \Sigma^{\mathrm{op}}$ be a functor satisfying 6.1(i). Then the category $\Sigma^{\mathrm{op}}$-sets (of functors $\Sigma^{\mathrm{op}} \rightarrow$ sets and natural transformations between them ) admits a closed model category structure in which a map $X \rightarrow Y \in \Sigma^{\mathrm{op}}$ sets is a weak equivalence or a fibration whenever the induced map $j^{*} X \rightarrow j^{*} Y \in \mathbf{S}$ is so and in which the cofibrations are the retracts of the (possibly transfinite) compositions of cobase extensions of the inclusions $\dot{\Sigma}[n] \rightarrow \Sigma[n](n \geqslant 0)$.

Similarly, if the category $\mathbf{S} / N \boldsymbol{\Sigma}^{\text {op }}$ of simplicial sets over $N \Sigma^{\text {op }}$ has the closed model category structure induced by the usual one on $\mathbf{S}$ [9, Chapter II], if $* \in \Sigma^{\text {op }}$-sets is the terminal object and if $L^{\Sigma^{\text {op }}}$-sets $\rightarrow \mathbf{S} / N \Sigma^{\text {op }}$ denotes the functor which sends an object $X \in \Sigma^{\text {op }}$-sets to the map (see 3.6)

$$
\operatorname{holim}^{\Sigma^{\mathrm{op}}} i X \rightarrow \operatorname{holim}^{\Sigma^{\mathrm{op}}} i *=N \Sigma^{\mathrm{op}},
$$

then the arguments of the proof of Theorem 5.1 yield

6.3 TheOREM. Let $\Sigma$ be a small category with connected nerve and let $j: \Delta^{\mathrm{op}} \rightarrow \Sigma^{\mathrm{op}}$ be a functor satisfying 6.1(i) and (ii). Then the functor $L^{\Sigma^{\circ p}}$ has as right adjoint the functor

$$
R^{\Sigma^{\mathrm{op}}}=\operatorname{hom}\left(L^{\Sigma^{\mathrm{op}}} \Sigma[-],-\right): \mathbf{S} / N \Sigma^{\mathrm{op}} \rightarrow \Sigma^{\mathrm{op}}-\text { sets }
$$

and this pair of adjoint functors satisfies the (see §4) equivalence conditions of Quillen.

6.4 Corollary. The categories $\Sigma^{\text {op }}$-sets and $\mathbf{S} / N \Sigma^{\text {op }}$ have equivalent homotopy theories in the strong sense that [5] their simplicial localizations with respect to the weak equivalences are weakly equivalent in the sense of $[5, \S 2]$. 
6.5 EXAMPLE. If in the definition of $\Lambda^{\mathrm{op}}$ (see 1.2(ii)) one omits the cyclic relations $\left(d_{0} s_{n+1}\right)^{n+1}=\mathrm{id}: \mathbf{n} \rightarrow \mathbf{n}(n \geqslant 0)$, one gets a category $\mathbf{K}^{\text {op }}$ which is a kind of covering of $\Lambda^{\mathrm{op}}$. There is an obvious functor $j: \Delta^{\mathrm{op}} \rightarrow \mathbf{K}^{\mathrm{op}}$ which satisfies 6.1(i) and (ii). Moreover, the nerve $N \mathrm{~K}^{\mathrm{op}}$ is contractible and the homotopy theory of $\mathbf{K}^{\mathrm{op}}$-sets thus is equivalent to the usual homotopy theory of simplicial sets.

6.6 EXAMPLE. Given a simplicial group $G$, one can form its flattening $\mathbf{b} G$, i.e. [7, §7] the category which has as objects the $\mathbf{n}(n \geqslant 0)$ and as maps $\mathbf{k} \rightarrow \mathbf{n}$ the pairs $(e, g)$, where $e$ is a map $e: \mathbf{k} \rightarrow \mathbf{n} \in \Delta^{\mathrm{op}}$ and $g$ is an $n$-simplex of $G$. There is an obvious functor $j: \Delta^{\mathrm{op}} \rightarrow \mathrm{b} G$ given by $j e=(e, 1)$ for all $e \in \Delta^{\mathrm{op}}$. Moreover, one readily verifies that this functor satisfies 6.1 (i) and (ii) and that $N \mathrm{~b} G$ is weakly equivalent to the classifying complex of $G$. Hence, the resulting homotopy theory of bG-sets is equivalent to the homotopy theory of simplicial sets over the classifying complex of $G$. This is, however, not surprising as $\mathbf{b} G$-sets are essentially the same as simplicial sets with a G-action.

We end with observing that in all three cases (i.e. $\Delta^{\mathrm{op}}, \mathbf{K}^{\mathrm{op}}$ and $\mathbf{b} G$ ) the functor $j$ was onto on objects. Actually this is not surprising in view of

6.7 Proposition. Let $\boldsymbol{\Sigma}$ be a small category with connected nerve and let $j$ : $\Delta^{\mathrm{op}} \rightarrow \Sigma^{\mathrm{op}}$ be a functor satisfying 6.1(i) and (ii). If $\boldsymbol{\Sigma}_{0}^{\mathrm{op}} \subset \Sigma^{\mathrm{op}}$ denotes the full subcategory spanned by the image of $j$, then the resulting functor $j_{0}: \Delta^{\mathrm{op}} \rightarrow \Sigma_{0}^{\mathrm{op}}$ also satisfies 6.1(i) and (ii) and the inclusion $\Sigma_{0}^{\mathrm{op}} \rightarrow \Sigma^{\mathrm{op}}$ induces an equivalence between the homotopy theory of $\boldsymbol{\Sigma}^{\mathrm{op}}$-sets and that of $\boldsymbol{\Sigma}_{0}^{\mathrm{op}}$-sets.

Proof. This follows immediately from Theorem 6.3 and Quillen's Theorem B [10, p. 97].

\section{BIBLIOGRAPHY}

1. K. S. Brown, Abstract homotopy theory and generalized sheaf cohomology, Trans. Amer. Math. Soc. 186 (1973), 419-458.

2. A. K. Bousfield and D. M. Kan, Homotopy limits, completions and localization, Lecture Notes in Math., vol. 304, Springer-Verlag, 1972.

3. A. Connes, Cyclic cohomology and functors Ext $^{n}$, C. R. Acad. Sci. Paris 296 (1983), 953-958.

4. E. Dror, W. G. Dwyer and D. M. Kan, Equivariant maps which are self homotopy equivalences, Proc. Amer. Math. Soc. 80 (1980), 670-672.

5. W. G. Dwyer and D. M. Kan, Function complexes in homotopical algebra, Topology 19 (1980), $427-440$.

6. Singular functors and realization functors, Nederl. Akad. Wetensch. Indag. Math. A87 $=$ Indag. Math. 45 (1984), 147-153.

7. __ Equivariant homotopy classification, J. Pure Appl. Algebra (to appear).

8. T. G. Goodwillie, Cyclic homotopy derivations and the free loop space, preprint.

9. D. G. Quillen, Homotopical algebra, Lecture Notes in Math., vol. 43, Springer-Verlag, 1967.

10. , Higher algebraic K-theory. I, Lecture Notes in Math., vol. 341, Springer-Verlag, 1973, pp. $85-147$.

Department of Mathematics, University of Notre Dame, Notre Dome, Indiana 46556

Department of Mathematics, Princeton University, Princeton, New Jersey 08544

Department of Mathematics, Massachusetts institute of Technology, Cambridge, MasSACHUSETTS 02139 